\title{
Research on the Effective Mechanism of Transforming Scientific Research Results of Business Administration to Teaching Case
} Wen-he LIN ${ }^{1}$, Wen-bao WANG ${ }^{2,}{ }^{*}$, Rong-rong $\mathrm{ZHAO}^{3}$ and Lu-bin KE ${ }^{4}$

\author{
${ }^{1,3,4}$ College of Management, Fujian agriculture and forestry university, Fuzhou, China
}

${ }^{2}$ School of Management, Fujian University of Technology, Fuzhou City, 350118, China.

${ }^{*}$ Corresponding author

Keywords: Business administration, Research results, Teaching Cases.

\begin{abstract}
The transformation of scientific research achievements of industrial and Commercial Administration into teaching cases is beneficial to combine the scientific research and teaching functions of teachers and improve the teaching quality of colleges and universities. The transformation of the scientific research achievements of Industry and Commerce into teaching cases is a feed-forward process. The scientific research achievements provide rich material for teaching cases. But the transformation process will be influenced by the educational policy, the teachers 'own ability, the teachers' evaluation mechanism and the transformation incentive mechanism. Therefore, we can guarantee the effective operation of the transformation mechanism from the scientific research achievements of Industry and Commerce to the teaching case by improving the policy guidance, the teachers 'understanding of the teaching case, the teachers' evaluation mechanism and the incentive mechanism.
\end{abstract}

\section{探索工商管理学科科研成果向教学案例转化的有效机制}

\author{
林榅荷 ${ }^{1}$ ，王文宝 ${ }^{2 *}$, 赵蓉蓉 ${ }^{3}$, 柯露涁 4 \\ 1,3,4福建农林大学管理学院, 福州, 中国 \\ 2福建工程学院管理学院, 福州, 中国 \\ “通讯作者
}

关键词: 工商管理学科; 科研成果; 教学案例

摘要: 工商管理学科科研成果转变为教学案例, 有利于将教师的科研职能与教学职能有机结 合, 提高高校的教学质量水平。工商管理学科科研成果向教学案例转化是一个互馈的过程, 科研成果为教学案例提供丰富的素材, 教学案例的应用也有利于进一步完善科研成果。但转 换过程会受到高校教育政策、教师自身能力、教师评价机制和转化激励机制的共同影响。因 此, 可以通过完善政策引导、提高教师对于教学案例的认识、完善教师评价机制建设、完善 激励机制建设来保障工商管理学科科研成果向教学案例转化机制的有效运转。

\section{1. 引言}

高校作为重要的科学研究力量, 在理论研究、专利研究、创新研发等方面发挥着重要的 作用, 源源不断的科研成果从高校走向各个领域, 发挥着不容忽视的作用。然是, 高校作为 社会人才的主要培养基地，教书育人同样是高校的重要使命，是高校教师不容忽视的重要“本 职工作”。但高校的评价机制导致高校教师更多将精力投入科研方面，虽然促进了科技创新以 及新知识的创造, 但一定程度上造成高校教师过分重视科研而轻视教学的现象。导致科研成 果脱离实际, 没有转化为课程教学资源为学生服务, 而高校中的学生也不能对最新的科研成 
果有深刻认识, 学习内容陈旧, 最终形成科学与教学脱节的情况, 教学与科研的结合仅仅停 留在形式上。吕文学 (2003) 指出, 如何加强科研成果与教学的粘性, 提升科研成果的实践 意义已经成为当今高校教学改革的当务之急 ${ }^{[1]}$ 。教学案例是高校中教学的重要工具, 可以充 分利用教学案例的优势, 将科研成果转化为教学资源, 并在教学过程中加深对科研成果的运 用于理解, 工商管理学科在高校中有着丰富的科研成果, 但是同样面临科研成果与教学脱离 的问题, 探索工商管理学科科研成果向教学案例转化机制, 一方面可以向高校教师将自身的 科研成果进行教学转化提供思路与指导; 另一方面也有利于丰富工商管理学科科研成果。

\section{2. 科研成果向教学案例转化的相关研究}

目前, 关于科研成果向教学转化的相关研究, 已经有机构以及高校对这一问题进行了研 究, 得出的研究成果主要围绕科研教学转化的意义、提升教学转化效果的措施以及方式的研 究。

第一, 科研向教学转化的意义。吴志强等 (2017) 指出高校教师将科研成果转化为教学 资源, 可以丰富课程内容, 拉进与学生之间的距离, 教师可以结合科研与教学的双重需求, 形成有自身特色的教学思维, 更好的发挥教书育人的作用 ${ }^{[2]}$ 。林建平等 (2015) 指出, 通过 案例研究方法将科研成果与教学统一为一个整体, 可以拉近理论与实践之间的距离, 提高研 究结论的情境化 ${ }^{[3]}$ 。夏泽等 (2017) 指出, 科研有助于学生科学学习理念的梳理, 从而形成 正确的科学思维, 并能提供有效的实际锻炼 ${ }^{[4]}$ 。

第二, 科研向教学转化的措施。李奋 (2011) 指出高校要促进科研成果与教学的有效融 合, 首先, 应强化科研、学科建设支持人才培养理念; 其次, 强化科研及其成果服务于专业 教学的理念; 再次, 运用同等激励机制促进科研向教学转化; 最后, 设计合理的教学、科研 考核机制, 促进人才培养 ${ }^{[5]}$ 。祁红岩等 (2016) 指出要提高科研成果向教学资源的转化, 首 先, 各高校要加强交流, 学习成果经验, 挖掘科研成果转化的内在驱动力; 其次, 建立学术 水平与教学水平兼备的教师评价机制; 最后, 有效解决科研与教学在时间与空间上的矛盾, 最终提升科研成果向教学内容有效转化 ${ }^{[6]}$ 。

第三, 科研成果向教学转化的方式。周智华等人（2017）以本科教学为基础展开研究, 认为高校教师科研成果转化为教学资源的方式多种多样, 其中, 将科研成果融入教学过程、 将科研成果设计为创新实验、引导学生参与科研创新、科学竟赛和自制仪器设备形式最为典 型 ${ }^{[7]}$ 。解璞等 (2015) 立足于研究生教育, 可以理论引申方式深化教学内容; 完善科研成果 表述形式, 以案例等方式丰富教学模式; 开设科研前沿成果专题, 共享科研资源 ${ }^{[8]}$ 。

虽然已经有部分学者展开了对科研成果向教学资源转化的相关研究, 也有少数学者在提 出措施中提到了教学案例与科研成果的结合方法, 但是还没有人系统的探索科研成果向教学 案例转化有效机制, 本研究以工商管理学科为学科基础, 填补了该方面研究的不足, 具有重 要的理论与实践意义。

\section{3. 科研成果向教学案例转化机制分析}

\section{1 影响工商管理学科科研成果向教学案例转化的因素}

\section{1、教师自身能力}

工商管理学科的科研成果主要来自于高校教师的研究, 将科研成果转化为教学案例工作 的开展也主要是教师的行为, 因此, 高校教师自身的能力高低直接影响工商管理学科科研成 果向教学案例转化的成效。教师的转化能力不足, 制约了工商管理学科科研成果向教学案例 转化成效。教师自身能力对于科研成果向教学案例转化机制的制约主要体现在两个方面: 第 一, 教师科研能力的欠缺。现今高校中许多教师科研能力不足, 缺乏创新精神, 甚至部分高 校教师长期科研工作缺位, 导致其难以产出高质量高水平的科研成果。此外, 部分工商管理 学科教师在研究选题时通常追求研究成果的学术性和先进性, 最终研究成果虽然具备了创新 
要求, 但忽视了成果向教学案例或者教学资源转化的适应性问题, 增加了科研成果向教学案 例转化难度, 降低最终的转化成效。第二, 高校教师科研成果向教学案例转化意识淡薄。目 前许多高校的教师对于科研成果向教学案例转化的认知观念不足, 功利看待科研成果, 忽视 将科研成果转化为教学案例的重要意义, 转化意识不强。

2、高校教育政策

将工商管理学科科研成果转化为教学案例同样需要教育政策引导，当有明确的教育政策 规定高校教师要注重科研成果转化时, 高校教师关于科研成果向教学案例转化的意识也会加 强, 进而影响教师的转化行为, 提升科研成果向教学案例转化的成效。但是, 目前很少有关 于工商管理学科科研成果向教学案例转化的相关教育政策, 这种政策缺失一方面导致教师成 果转化意识薄弱, 另一方面在将科研成果转化为教学案例时没有政策依据, 仅能依靠教师个 人经验和主观判断, 导致最终呈现结果层次不齐, 没有标准, 整体转化效果不佳。此外, 由 于科研成果向教学案例转化相关教育政策缺失, 导致转化的保障体系不健全。一方面, 成果 转化没有政策的引导, 也没有相应的转化标准, 导致科研成果向教学案例转化工作成为可有 可无的口号, 最终流于形式, 无法落实。另一方面, 在学校财政资金方面没有足够的支持, 导致教师在将工商管理学科科研成果转化为教学案例时没有充足的资金保证, 缺乏健全保障, 阻碍了转化工作开展。

3、教师评价机制

教师的评价机制是教师开展工作的重要依据, 对于工商管理学科科研成果向教学案例专 化的相关评价内容可以激发教师探索转化的动力, 也为教师的转化工作提供了一定依据。现 今高校中的教师评价机制主要是对教师的科研和教学两方面的评价, 但是关于科研成果转化 的相关评价内容欠缺, 导致教师往往将科研与教学分割开, 忽视二者之间的内在联系。评价 机制的欠缺主要表现在以下几个方面：第一，评价内容不完整。现有关于科研成果转化为教 学资源的评价指标内容较宏观, 很少有专门针对科研成果向教学案例转化的评价内容, 加之 科研成果种类较多, 转化形式多样, 很难客观全面地评价转化行为。很多高校还停留在只要 有将科研成果转化为教学资源的行为即可的阶段, 重视程度严重不足。第二, 评价标准欠缺。 将工商管理学科科研成果转化为教学案例的转化结果存在的可能性较多, 不同教师的最终转 化效果差异性较大, 相应的评价标准需要保证科学性和细致性。许多高校对于科研成果转化 为教学案例的具体评价标准欠缺, 一方面导致教师在开展转化工作时没有准确的依据; 另一 方面导致学校在对教师的转化工作展开评价过程的随意性与不合理性。

4、转化激励机制

高校对于教师将工商管理学科科研成果转化为教学案例的激励机制, 很大程度上会影响 教师的转化工作热情与积极性, 因此, 在科研成果转化为教学案例的机制中, 激励机制的健 全与否很大程度上会影响最终的转化效果。但是, 目前高校对于科研成果转化的激励机制, 激励程序严重欠缺。第一, 激励目标缺失。有效科学的激励目标可以对教师的行为产生正向 的引导, 激发其实现目标的热情与自主性。目前, 高校很少有针对将科研成果转化为教学案 例的激励政策, 而对于转化的实际目标要求更少, 导致很多教师缺乏成果转化的热情。第二, 评估工作不健全。高校在对教师进行考核以及相应的评定职称时，往往只将关注点放在教师 发表论文的数量和等级上, 很少有将科研成果转化为教学案例的情况纳入教师考评范围, 相 应的也没有一套完整的关于科研成果转化为教学案例的评级体系, 造成工商管理学科领域内 将科研成果转化教学案例的成果稀少。此外, 评估的最终结果也没有设置相应的奖罚机制, 即使有部分教师自主将科研成果转化为教学案例, 这些转化者也得不到相应的奖励, 打击了 他们对于科研成果转化的积极性, 不利于科研成果转化机制的进一步完善。

\section{2 工商管理学科科研成果转化为教学案例的机制分析}

工商管理学科科研成果转化为教学案例是一个互馈的过程 (具体情况如图 1 所示)。

第一，教师通过工商管理领域内的实践现象进行实践研究，再根据自身的理论知识储备 和相应的研究方法, 对实际的管理问题进行系统的理论化处理, 最终得出工商管理学科的科 
研成果。这些科研成果为管理教学案例提供了丰富的素材, 是教学案例非常重要的来源。教 师对科研成果有着深刻的认识与理解, 且其对学生教学也有着熟练的经验和深刻的认识, 这 种双重身份可以有效保障科研成果转化为教学案例的有效性。第二, 由科研成果转化来的教 学案例又可以反馈科研实践, 进一步完善教学成果。在教学的过程中, 教师在进行案例教学 时可以从学生处得到该科研内容的思考反馈, 并能收集到丰富的数据, 为进一步完善科研成 果提供实践基础和检验思路。第三, 在工商管理学科科研成果转化为教学案例的互馈过程中, 会受到高校教育政策、教师自身能力、教师评价指标以及转化激励机制的共同影响, 最终形 成完整的转化机制。高校的教育政策从宏观层面引导教师将科研成果转化为教学案例, 并为 其提供相应的保障机制。教师自身的能力决定了科研成果以及教学案例转化的水平。教师评 价指标为教师将科研成果转化为教学案例提供指导与标准。转化激励机制影响教师将科研成 果转化为教学案例的内在动力。

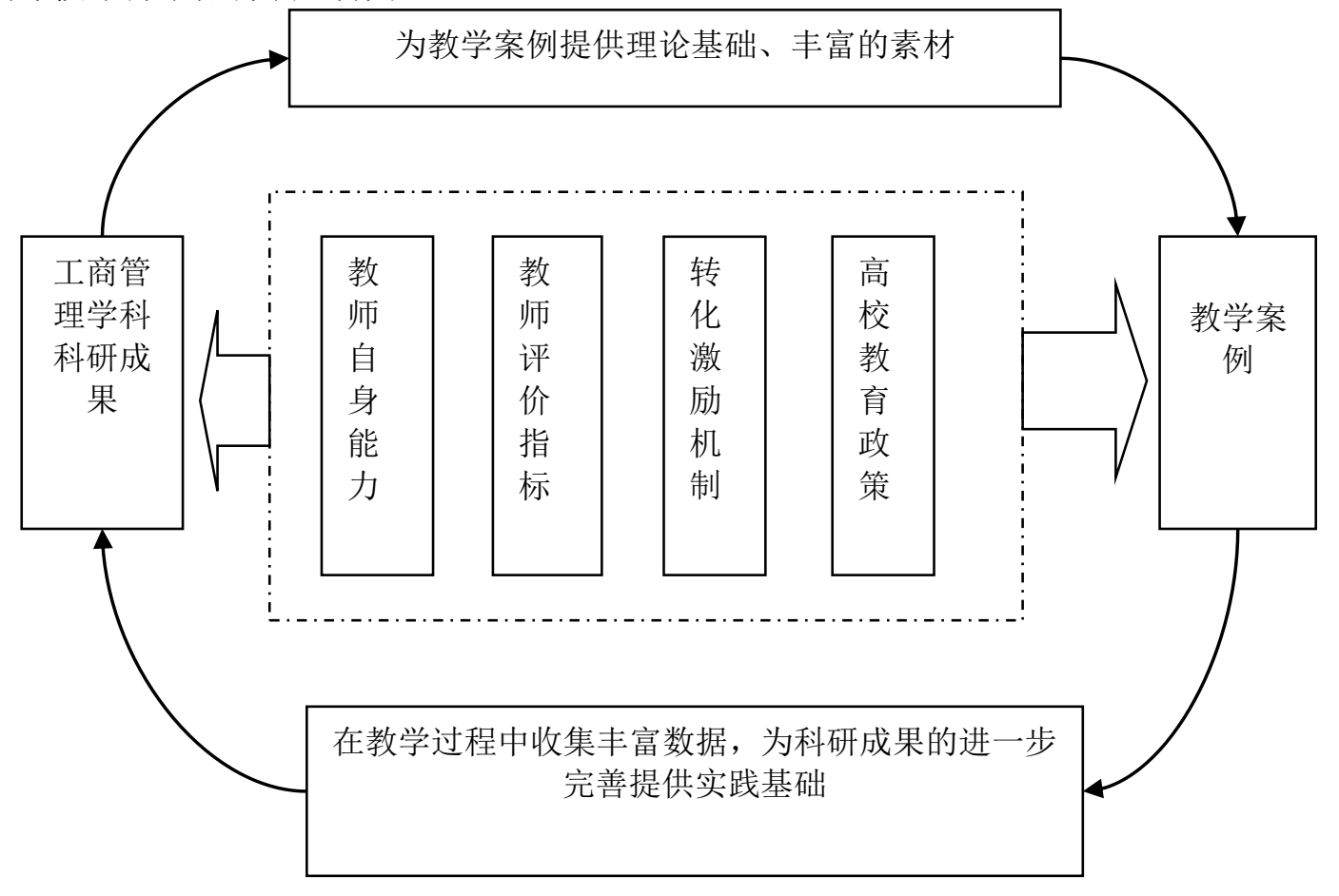

图1 工商管理学科科研成果向教学案例转化机制图

\section{4. 工商管理学科科研成果向教学案例转化机制的保障措施}

\section{1 完善政策引导}

第一，制定科研成果向教学案例转化政策。各高校应以现有科研成果向教学资源转化的 教育政策为导向, 制定有自身特色的工商管理学科科研成果向教学案例转化的细分政策与制 度, 促进科研成果积极向教学案例转化, 为教师的成果转化工作提供政策引导与制度支持, 将加快科研成果向教学资源转化的步伐。

第二, 改革体制, 提供支持。高校要改变以往科研与教学分开的管理体制, 重视科研成 果向教学案例转化, 并加强管理, 提供充分的支持。一方面, 高校要制定体制和制度规定, 下发相关的文件, 明确科研成果向教学案例转化的重要性和相关标准, 从政策文件中给予保 障, 为教师开展转化工作以及管理部门开展管理工作提供制度标准, 和制度依据。其次, 将 科研成果向教学案例转化工作纳入教学和科研补贴范围内, 为其提供充分的资金支持, 减少 转化阻碍的同时激发教师的转化热情和转化工作开展的信心。 


\section{2 提高教师对于教学案例的认识}

第一，提高教师科研能力。教师应该注重对自身科研能力的提升，积极参与到科研创新 工作中去, 努力创造成更多的成果, 保障教学案例的转化来源。此外, 教师也应该有意识的 培养自身将科研与教学结合的能力, 在实际的教学工作中锻炼转化能力, 高校可以为教师提 供提升的平台和培训机会。

第二, 转变观念。转变以往“重学术, 轻教学”的观念, 不断强化科研成果转化意识。此 外, 高校教师在进行科研选题时也要对选择的教学意义进行提前预判, 管理科学领域的教师 要将科研成果转化为教学案例, 将科研成果运用到实际的教学工作中看做是工作的一部分, 转化工作视为己任, 最终在整个学科领域内形成学科成果转化与延伸的氛围。

\section{3 完善教师评价机制}

高校应注重对工商管理学科科研成果向教学案例的转化, 完善转化的评价机制, 进一步 优化教学案例转化机制。

第一, 完善转化评价内容。首先, 明确工商管理学科的特点, 将科研和教学评价体系有 机结合, 将科研成果转化为教学案例纳入教师绩效考核的范畴中去, 提高教师对于科研成果 转化的重视程度。其次, 将科研成果转化效果作为评价内容。将科研成果向教学案例转化的 最终效果作为评价内容, 避免转化工作流于形式, 增强教师的成效意识, 从而提升转化的效 果。

第二，优化评价标准。针对工商管理学科科研成果向教学案例转化的需要，将转化工作 划分为不同的环境和模块, 制定一套严格的评价标准, 采用量化评价指标体系, 依据转化的 工作投入以及成效给予相应的分值, 最终将所有分值相加作为科研成果向教学案例转化工作 评价得分。

第三，优化评价方式。学生的态度以及学生的收获最能体现转化的成效。高校在对教师 进行评价时可以将学生的看法和学生的成长纳入考量范围, 作为科研成果向教学案例转化成 效的参考标准, 提高评价的科学性与客观性。

\section{4 完善激励机制建设}

第一, 明确科研目标。高校的领导应转变传统的科研观念, 重新考量科研与教学的关系, 强调科研成果向教学案例转化的重要性, 进而将正确的科研观念向教师传达, 树立可以向教 学转化的科研目标。从而激励教师在开展科研工作时, 注重选题向教学转化的可能性, 激发 教师自主推进科研成果向教学案例转化目标的实现, 实现科研成果向教学案例转化机制的有 效运转。

第二, 将评价结果与奖励挂钩。将科研成果转化为教学案例纳入奖励政策范畴, 对于科 研成果向教学案例转化成效高的教师给予奖励。此外, 要加强科研成果向教学案例转化的评 价结果与奖励的联系, 并且可以将评价结果与教师的职称评定、薪酬福利提升挂钩。将科研 成果向教学案例转化与教师的直接利益联系在一起，从而激发教师转化的动力。

\section{5. 结束语}

研究工商管理学科科研成果向教学案例转化的机制, 可以为高校教改提供思路, 改变现 有学术与教学分离的现状。目前学术界已经部分学者对科研成果向教学资源转化展开了研究, 但是还没有关于工商管理学科科研成果向教学案例转化机制的研究。本文以工商管理学科为 研究对象, 分析其科研成果向教学案例转化的内在机制, 发现科研成果向教学案例转化是一 个互馈的系统, 这一运行机制会受到教育政策、教师自身能力、教师评价指标和转化激励机 制四个方面因素的影响。因此, 提出, 可以通过完善政策引导、提高教师对于教学案例的认 识、完善教师评价机制建设、完善激励机制建设来保障工商管理学科向教学案例转化机制的 有效运转。 


\section{致谢}

本文为福建省社科研究基地重大项目 (2016JDZ039) 、福建农林大学杰出青年科研人才 计划项目（xjq201636）、2017年度 “福建省高校杰出青年科研人才培育计划”项目、2017年 福建农林大学科技创新专项基金项目(CXZX2017405)的阶段性成果之一。

\section{References}

[1] Wen-xue Lv,Li-han Zhang,Yan-ru Zhang, A Study on the Two-way Driven Teaching Practice of Scientific Research Teaching, J. Education exploration,4(2013)38-40.

[2] Zhi-qiang Wu, Ai-ming Chu, Li-wei Lu, Reflections on the Transformation of Scientific Research Achievements into Teaching Resources in Undergraduate Teaching Auditing Evaluation, J. Contemporary Educational Theory and Practice, J. Contemporary Educational Theory and Practice, $1(2017) 65-67$.

[3] Jian-ping, Mian-zhen Pan, Integration of scientific research teaching based on case study: mechanism, experience and measures, Personnel Training and Teaching Reform, Hangzhou, 2015.

[4] Ze Xia, Tao chen, Xiao-fang Zhao, Qian-qian Tang, The clinical research results in the teaching of support for clinical medicine professional construction, J. Popuhr Science\&Technology, 215(2017)132-133.

[5] Fen Li, Reflections on the Relationship between Scientific Research and Teaching in University Teachers ,J. Journal of Jinhua College of Profession and Technology, 2(2011)23-26.

[6] Hong-yan Qi, Jie Xu, Strategy Research on Transforming Scientific Research Achievements into Teaching Resources ,J. Heilongjiang Education, 1(2016)6-7.

[7] Zhi-hua Zhou, Guo-bin Li, An-ping Tang, Li-hua Liu, Guo-rong Xu, University teachers scientific research into undergraduate teaching resources form, J. Contemporary Educational Theory and Practice, 6(2017)105-107.

[8] Pu Jie, Jin-cheng Zhao, Yi Liu, Study on Transformation of Scientific Research Achievements into Postgraduate Teaching Resources ,J. Economic Management,11(2015)261-262. 